\title{
Research on Preheating Temperature Field Test of Natural Gas Pipeline in Service Welding under Extreme Condition
}

\author{
Yan Xu ${ }^{l,}{ }^{*}$, Yinglai Liu ${ }^{l}$, Zhenjun Feng ${ }^{l}$, Xianghui Nie ${ }^{1}$, Chao Zhang ${ }^{1}$, Jasmine ${ }^{2}$ \\ ${ }^{1}$ Tubular Goods Research Institute of CNPC, Xi'an, China \\ ${ }^{2}$ Companhia Brasileira de Metalurgia e Minerao, Brazil
}

\begin{abstract}
When the natural gas pipeline is welding in service, the fast flowing medium with pressure in the pipe will take away a lot of heat, and the preheating temperature is not easy to be guaranteed, so it is easy to appear hydrogen-induced crack. In this paper, the in-service welding preheating temperature field of natural gas pipeline under the limit condition of unreduced volume was studied, and the pre-welding preheating test was carried out by using the medium frequency heating method. It is found that the temperature below the heating belt increases gradually with the increase of the intermediate frequency heating power, and the fitting shows a quadratic polynomial gradient. There are differences in preheating temperatures on the same circumference. The highest temperature mostly appears in the direction of 3 point of the pipeline, while the lowest temperature mostly appears in the direction of 0 point, which is related to the tightness of the heating belt, sunshine, blowing and other factors. In addition, the preheating temperature field of the pipeline in service is related to the gas flow in the pipeline. At the same heating power, the downstream temperature of the heating belt is higher than the upstream temperature at the same location, and the closer to the heating belt, the higher the temperature is. When the gas flow rate reaches $9.37 \mathrm{~m} / \mathrm{s}$ and the heating power is $160 \mathrm{~kW}$, the average measured temperature at $50 \mathrm{~mm}$ upstream and downstream of the heating belt of $\Phi 1016$ pipeline is $107^{\circ} \mathrm{C}$, and the average measured temperature at $50 \mathrm{~mm}$ upstream of the heating belt is $71^{\circ} \mathrm{C}$. When the gas flow rate reaches $8.91 \mathrm{~m} / \mathrm{s}$ and the heating power is $200 \mathrm{~kW}$, the average measured temperature at the downstream $50 \mathrm{~mm}$ of the heating belt of $\Phi 1219$ pipeline is $72^{\circ} \mathrm{C}$, the average measured temperature at the upstream $50 \mathrm{~mm}$ of the heating belt is $52^{\circ} \mathrm{C}$ and the average measured temperature at the upstream $30 \mathrm{~mm}$ of the heating belt is $71^{\circ} \mathrm{C}$..
\end{abstract}

\section{Introduction}

As an important means of in-service welding repair, B sleeve repair technology can not only ensure continuous pipeline transportation, but also reduce the degree of environmental damage, shorten the repair cycle and reduce the repair cost [1-2]. The repair technology is applicable to a wide range of defects, including pipeline corrosion, cracks, mechanical damage, weld defects, etc., and can also repair leakage defects, with good repair effect and high reliability, which belongs to permanent repair[3-4].When the B-type sleeve is welded, the pipeline is in an uninterrupted state, and there is a rapid flow of pressurized gas or liquid medium in the pipeline, which is difficult to ensure the preheating temperature and interlayer temperature. At the same time, the cooling rate of weld is too fast, which will promote the formation of hydrogen-induced crack-sensitive harden structure in welded joints, so hydrogen-induced cracks are easy to occur and the bearing capacity of the joints is reduced. [56].Preheating before welding can slow down the cooling rate after welding, and promote the diffusion of hydrogen, water and other pollutants. It can not only avoid the generation of hydrogen-induced cracks, but also reduce the hardening degree of weld and heat affected zone, and improve the crack resistance of welded joints [7-11]. At present, the commonly used preheating methods before welding are flame heating and intermediate frequency heating, but the preheating temperature often cannot meet the welding requirements in the construction. The pipeline to be repaired needs to reduce the flow rate of the conveying medium. Therefore, it is necessary to study the relationship between the preheating temperature of the inservice welding of the natural gas pipeline and the heating power and the flow rate of the medium, especially the distribution of the preheating temperature field of the inservice welding of the natural gas pipeline under the extreme working conditions.

\section{Testing method}

Taking the West-East Gas Pipeline as the research object, several gas pipelines with $\Phi 1016 \mathrm{~mm}$ (wall thickness $14.6 \mathrm{~mm}$, pipe flow rate $4.60 \sim 9.82 \mathrm{~m} / \mathrm{s}$ ) and $\Phi 1219 \mathrm{~mm}$

*Corresponding author e-mail: xuyan008@cnpc.com.cn 
(wall thickness $15.3 \mathrm{~mm}$ or $18.4 \mathrm{~mm}$, pipe flow rate $7.51 \sim 8.91 \mathrm{~m} / \mathrm{s}$ ) in service were excavated, and the anticorrosive layer was stripped. The pipe body was heated by medium frequency heating method, and the temperature distribution below and upstream and downstream of the medium frequency heating zone was recorded by thermocouple real-time test (Figure 1, the temperature test point number below the heating zone was $\mathrm{P} 1 \sim \mathrm{P} 16$, and the temperature test point number above the heating zone was B1 B16). The temperature tracking was carried out at the test point $50 \mathrm{~mm}$ away from the edge of the heating zone ( the farthest welding channel position during B sleeve welding ). The heating test was carried out by using several intermediate frequency heating equipment with rated power commonly used in welding. Different initial power was set according to different equipment. After holding for $10 \mathrm{~min}$, it was increased by $20 \mathrm{~kW}$ in turn. The heating was stopped when the preheating temperature at the farthest weld position reached the welding requirement $\left(60^{\circ} \mathrm{C}\right)$ or the equipment power reached the limit.

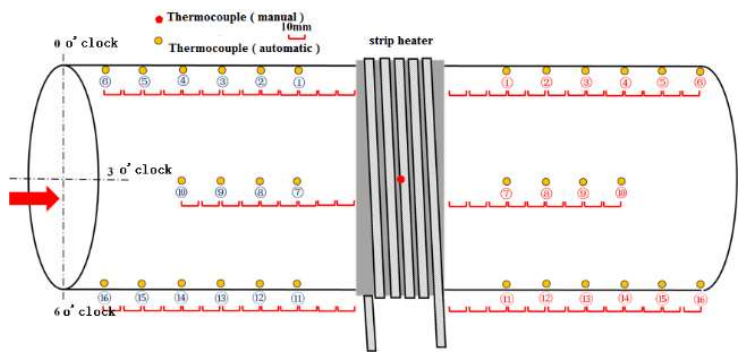

Figure 1. Layout of temperature test points

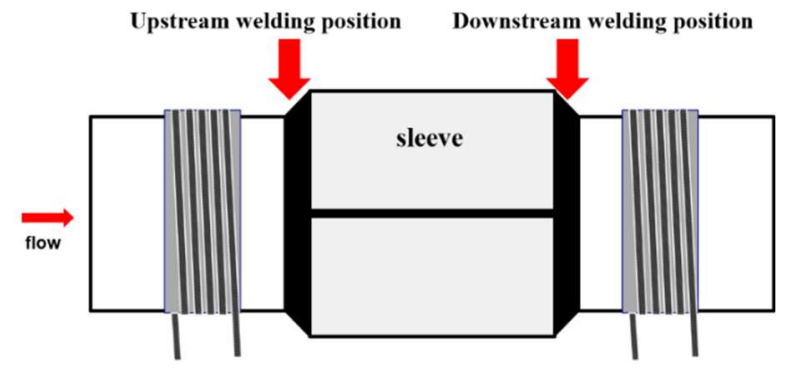

Figure 2. B sleeve welding schematic

\section{Test results and analysis}

\subsection{Test temperature below tropical zone}

The test temperatures of $\Phi 1016 \mathrm{~mm}$ pipeline and $\Phi 1219 \mathrm{~mm}$ pipeline with P1 P16 below the tropical zone under different heating power are summarized respectively (Figure 3 ). It can be seen that the temperature below the tropical zone of the pipeline and the heating power of the intermediate frequency equipment show a quadratic polynomial gradual change. With the increase of the heating power of the intermediate frequency equipment, the temperature below the tropical zone increases gradually.

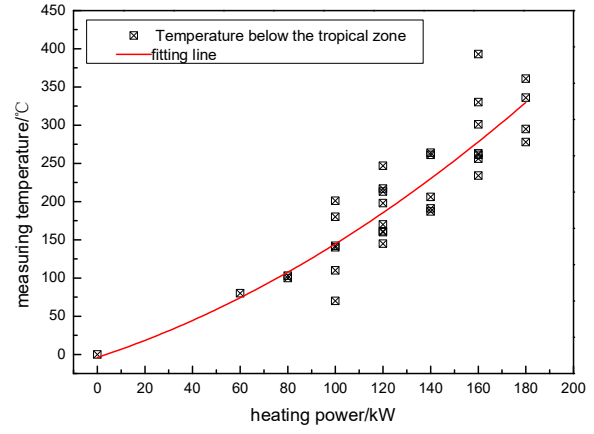

(a) $\Phi 1016 \mathrm{~mm}$ pipeline

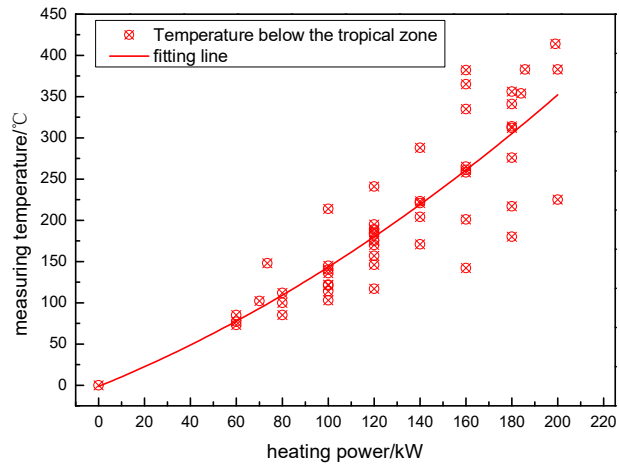

(b) $\Phi 1219 \mathrm{~mm}$ pipeline

Figure 3. The relation curve between the test temperature and the intermediate frequency heating power under the heating zone of different pipe diameters

\subsection{Upper and Lower Tropical Temperatures}

The heating temperature measurement of $\Phi 1016 \mathrm{~mm}$ pipeline under the condition of pressure $8 \mathrm{MPa}$, flow rate $2850 \times 104 \mathrm{~m} 3 / \mathrm{d}$ and flow rate $4.60 \mathrm{~m} / \mathrm{s}$ was analyzed, and the temperature distribution and temperature difference curve of the upstream and downstream of the heating zone under different heating power were obtained ( Figure 4 ). It can be seen that when the heating power is $180 \mathrm{~kW}$, the temperature below the tropical reaches $352^{\circ} \mathrm{C}$, the upstream and downstream are closer to the edge of the tropical, the higher the temperature, the downstream temperature is significantly higher than the upstream temperature with equal spacing, and the maximum temperature difference is close to $40^{\circ} \mathrm{C}$.

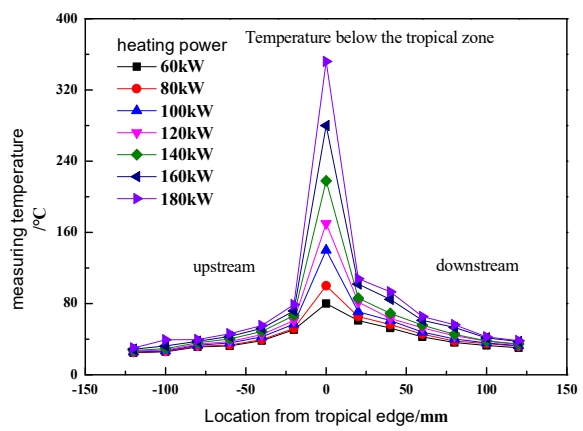

(a) Temperature distribution upstream and downstream 


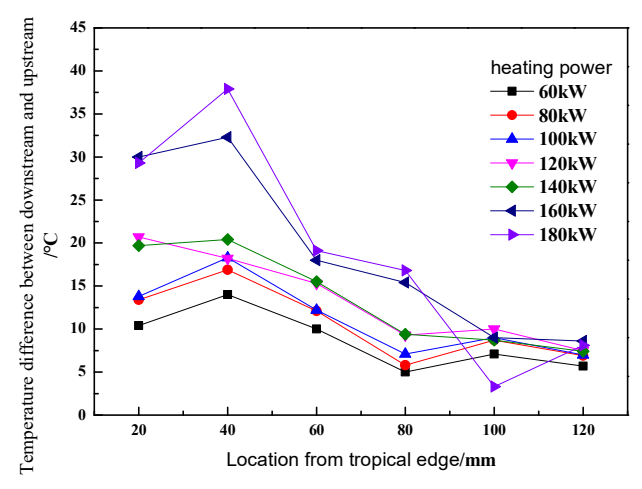

(b) Temperature difference upstream and downstream

Figure 4. Temperature distribution and temperature difference curves of the heating zone upstream and downstream under different heating powers of $\Phi 1016 \mathrm{~mm}$ pipeline

\subsection{Different quadrant temperature tests}

Under the ideal heating condition, the temperature distribution on the same circumference of the pipeline should be consistent, but in the actual measurement, it is found that there are differences in the data of 0,3 and 6 points on the same circumference of the tropical zone, so as to ensure that other basic conditions remain unchanged. The temperature of $\Phi 1016 \mathrm{~mm}$ pipeline and $\Phi 1219 \mathrm{~mm}$ pipeline in different quadrants are tested respectively, and the temperature test results of different quadrants in the heating test of the pipeline under the limit working condition of the West-East Gas Pipeline are obtained ( Figure 5 ). It can be seen that the maximum temperature mostly appears in the direction of 3 points, and the minimum temperature mostly appears in the direction of 0 points, which is related to the factors such as tropical winding tightness, sunshine and blowing. It is suggested that the heating effect should be improved as much as possible, and the heating coil should be tightly wrapped, especially at $6 \mathrm{o}$ ' clock. Building windbreaks when necessary.

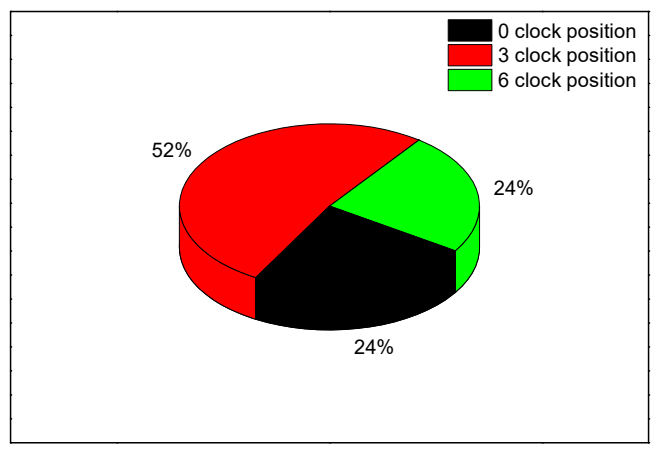

(a) The position of the clock at the highest temperature

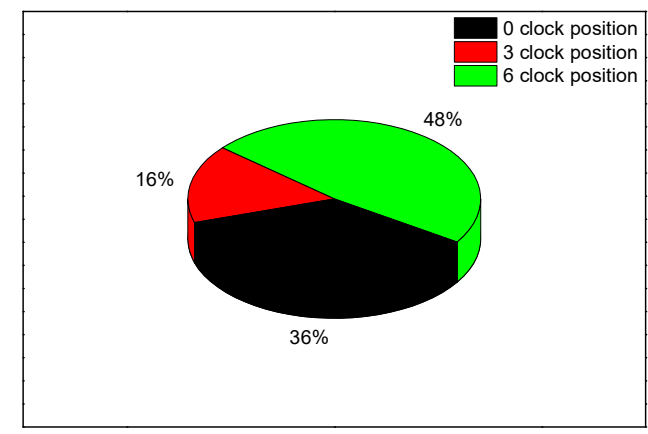

(b) The position of the clock at the lowest temperature

Figure 5. Test results of different quadrant temperatures for heating test of West-East Gas Pipeline

\subsection{Analysis of preheating temperature of pipeline in-service welding under extreme conditions}

According to the field feedback, the maximum gas flow rate of $\Phi 1016$ pipeline test section is $7-8 \mathrm{~m} / \mathrm{s}$ under normal operation, and the maximum gas flow rate of $\Phi 1219$ pipeline test section is $9-10 \mathrm{~m} / \mathrm{s}$ under normal operation. In this test, the maximum gas flow rate of $\Phi 1016$ pipeline reaches $9.37 \mathrm{~m} / \mathrm{s}$, and the maximum gas flow rate of $\Phi 1219$ pipeline reaches $8.91 \mathrm{~m} / \mathrm{s}$, which is close to or reaches the limit flow rate of the pipeline. Table 1 and table 2 are the test results of in-service welding preheating temperature of $\Phi 1016$ pipeline and $\Phi 1219$ pipeline under extreme conditions. The preheating temperature field of the in-service pipeline is related to the gas flow in the pipeline. Under the same heating power, the downstream temperature of the tropical zone is higher than the upstream temperature at the same position, and the closer to the tropical zone, the higher the temperature. When the gas flow rate reaches $9.37 \mathrm{~m} / \mathrm{s}$, under the heating power of 160 kilowatts, the measured average temperature at $50 \mathrm{~mm}$ downstream of the $\Phi 1016$ pipeline plus the tropical zone is $107^{\circ} \mathrm{C}$, and the measured average temperature at $50 \mathrm{~mm}$ upstream of the tropical zone is $71^{\circ} \mathrm{C}$, which basically meets the preheating temperature $\left(60^{\circ} \mathrm{C}\right)$ required by the current site. When the gas flow rate reaches $8.91 \mathrm{~m} / \mathrm{s}$, under the heating power of $200 \mathrm{~kW}$, the measured average temperature at $50 \mathrm{~mm}$ downstream of the $\Phi 1219$ pipeline heating tropical zone is $72^{\circ} \mathrm{C}$, and the measured average temperature at $50 \mathrm{~mm}$ upstream of the heating tropical zone is $52^{\circ} \mathrm{C}$. Among them, the preheating temperature at $50 \mathrm{~mm}$ upstream does not meet the requirements of welding preheating, while the measured average temperature at $30 \mathrm{~mm}$ upstream of the heating tropical zone is $71^{\circ} \mathrm{C}$. Therefore, it is recommended that the Btype sleeve should be installed in the $\Phi 1219$ pipeline under this condition, and the methods simultaneous heating of upstream and downstream, moving heating tropical zone and auxiliary heating should be used to meet the requirements of welding preheating. 
Table 1. In-service welding preheating temperature test results of $\Phi 1016 \mathrm{~mm}$ pipeline and $\Phi 1219 \mathrm{~mm}$ pipeline under different equipment output conditions under limit conditions

\begin{tabular}{|c|c|c|c|c|c|c|}
\hline \multirow{2}{*}{$\begin{array}{c}\text { pipe } \\
\text { diameter } \\
/ \\
\mathrm{mm}\end{array}$} & \multirow{2}{*}{$\begin{array}{c}\text { flow } \\
\text { velocity } \\
/ \\
\left(\mathrm{m} \cdot \mathrm{s}^{-1}\right)\end{array}$} & \multirow[b]{2}{*}{$\begin{array}{l}\text { heating } \\
\text { power } \\
/ \mathrm{kW}\end{array}$} & \multicolumn{4}{|c|}{$\begin{array}{l}\text { Measured average temperature } \\
\qquad{ }^{\circ} \mathrm{C}\end{array}$} \\
\hline & & & $\begin{array}{l}\text { Under } \\
\text { the } \\
\text { tropical } \\
\text { zone }\end{array}$ & $\begin{array}{l}\text { Upper } \\
\text { tropics } \\
50 \mathrm{~mm}\end{array}$ & $\begin{array}{l}\text { Upper } \\
\text { tropics } \\
30 \mathrm{~mm}\end{array}$ & $\begin{array}{c}\text { Lower } \\
\text { tropical } \\
\text { zone } \\
50 \mathrm{~mm} \\
\end{array}$ \\
\hline \multirow{5}{*}{1016} & \multirow{5}{*}{9.37} & 80 & 103 & 49 & 82 & 66 \\
\hline & & 100 & 172 & 56 & 99 & 80 \\
\hline & & 120 & 212 & 59 & 106 & 88 \\
\hline & & 140 & 310 & 69 & 135 & 103 \\
\hline & & 160 & 330 & 71 & 145 & 107 \\
\hline \multirow{6}{*}{1219} & \multirow{6}{*}{8.91} & 100 & 145 & 50 & 48 & 50 \\
\hline & & 120 & 183 & 53 & 52 & 54 \\
\hline & & 140 & 221 & 49 & 56 & 58 \\
\hline & & 160 & 265 & 52 & 60 & 66 \\
\hline & & 180 & 312 & 53 & 66 & 69 \\
\hline & & 200 & 383 & 57 & 71 & 72 \\
\hline
\end{tabular}

\section{Conclusion}

(1) The temperature below the heating band gradually increases with the increase of the heating power of the intermediate frequency equipment, and the fitting shows a quadratic polynomial gradual change.

(2) The preheating temperature on the same circle is different, which is related to the tropical winding tightness, sunshine, blowing and other factors. Among them, the highest temperature appears in the direction of 3 points, and the lowest temperature appears in the direction of 0 points. It is suggested that the heating effect should be improved as much as possible, and the heating coil should be tightly wrapped, especially at 6 o ' clock. Building windbreaks when necessary.

(3) The preheating temperature field of the in-service pipeline is related to the gas flow in the pipeline. Under the same heating power, the downstream temperature of the tropical zone is higher than the upstream temperature at the same position, and the closer to the tropical zone, the higher the temperature. When the gas flow rate reaches $9.37 \mathrm{~m} / \mathrm{s}$ and the heating power is $160 \mathrm{~kW}$, the measured average temperature of $\Phi 1016$ pipeline with $50 \mathrm{~mm}$ downstream of the tropical zone is $107^{\circ} \mathrm{C}$, and the measured average temperature of $50 \mathrm{~mm}$ upstream of the tropical zone is $71^{\circ} \mathrm{C}$. The preheating temperature basically meets the requirements of the current site $\left(60^{\circ} \mathrm{C}\right)$. When the gas flow rate reaches $8.91 \mathrm{~m} / \mathrm{s}$ and the heating power is 200 kilowatts, the measured average temperature of $\Phi 1219$ pipeline at $50 \mathrm{~mm}$ downstream of the tropical zone is $72^{\circ} \mathrm{C}$, the measured average temperature at $50 \mathrm{~mm}$ upstream of the tropical zone is $52^{\circ} \mathrm{C}$, and the measured average temperature at $30 \mathrm{~mm}$ upstream of the tropical zone is $71^{\circ} \mathrm{C}$. It is. It is suggested that the B-type sleeve should be installed under this condition, and the methods of simultaneous heating of the upstream and downstream, moving the tropics, and auxiliary heating should be used to meet the requirements of welding preheating.

\section{References}

1. WU Linjun. Reliability Analysis of Repair Technology of Pressure Pipeline in Service [J]. Quality and Technical Supervision Research, 2020(06):33-36.

2. HAO Jianbin, WU Xinjuan et al. In-Service Pipeline Repair and Simulation Testing Method of Emergency repair and Welding Process of Pipeline. OGST, 2006(02):37-40+62+2.

3. XU Minxu, LUO Lihui, ZHOU Huiping, et al. Influence of B -type Sleeve Welding on the Performance of X80 Pipeline Steel [J]. Materials Protection, 2019,52(06):149-156.

4. Li Rongguang, et al: Improvement on Remediation Technology for Type B Full-Encirclement Steel Sleeves. Oil \& Gas Storage and Transportation, 2010,29(10):755-758+717.

5. CHEN Juan, JI Feng, LIU Xuebin, et al. Control measures of in-service pipeline welding hydrogeninduced crack under low temperature environment [J]. Oil \& Gas Storage and Transportation, 2014, 33(12):1297-1300.

6. FU Wei, LYU Yuling. Burn-though and Hydrogen Induced Cracking Prediction of Oil and Gas Pipeline during In-service Welding [J]. Hot Working Technology,2018,47(23):241-245.

7. LI Weiwei, HE Xiaodong, GE Jialin. Enlightenment and Reference of Foreign Advanced Standards for Girth Welding of Oil and Gas Pipelines [J]. Petroleum Tubular Goods \& Instruments, 2020, 6(02): 1-7.

8. LI Weiwei, MA Qiurong, ZHAO Xinwei, et al. Effect of Pre-heating Temperature at Welding Heat Affected Zone of X80 PipelineSteel [J]. Petroleum Engineering Construction, 2005(04):10-12+4.

9. HE Xiaodong, WANG Yuexia, WU Shuang, et al.. Effect of Pre-heat Temperature and Cooling Rate on Properties in Coarse

10. Grain Heat-affected Zone for X80 High Deformability Pipeline Steel [J]. Welded Pipe and Tube,2012,35(10):10-15.

11. XU Yan, LIU Ying-lai, FENG Zhen-jun, et al.. Effect of Heat Treatment Temperature on Mechanical Tensile Properties of X80 Pipeline Steel [J]. Heat Treatment Technology and Equipment ,2020, 41(06):37-41.

12. Liu Yinglai, Wang Gaofeng, Nie Xianghui, et al..Influence of Normalizing Temperature on Microstructure and Mechanical Properties of Controlled Rolling Steel for Preparation of Manifolds [J]. Natural Gas and Oil, 2015,33( 